\title{
NPDD model: A tool for photopolymer enhancement.
}

\author{
Michael R. Gleeson ${ }^{1}$, Jinxin Guo ${ }^{2}$, Shui Liu $^{2}$ and John T. Sheridan ${ }^{2}$ \\ ${ }^{1}$ Department of Computer Science, National University of Ireland Maynooth, \\ Maynooth, Co. Kildare, Republic of Ireland. \\ ${ }^{2}$ UCD School of Electrical, Electronic and Mechanical Engineering, \\ College of Engineering, Mathematical and Physical Sciences, \\ Communications and Optoelectronic Research Centre, \\ SFI Strategic Research Centre in Solar Energy Conversion, \\ University College Dublin, Belfield, Dublin 4, Republic of Ireland.
}

\begin{abstract}
The use of theoretical models to represent the photochemical effects present during the formation of spatially and temporally varying index structures in photopolymers, is critical in order to maximise a material's potential. One such model is the Non-local Photo-Polymerization Driven Diffusion (NPDD) model. Upon application of appropriate physical constraints for a given photopolymer material, this model can accurately quantify all major photochemical processes. These include i) non-steady state kinetics, (ii) non-linearity iii) spatially non-local polymer chain growth, iv) time varying primary radical production, v) diffusion controlled effects, vi) multiple termination mechanisms, vii) inhibition, (viii) polymer diffusion and ix) post-exposure effects. In this paper, we examine a number of predictions made by the NPDD model. The model is then applied to an acrylamide/polyvinylalcohol based photopolymer under various recording conditions. The experimentally obtained results are then fit using the NPDD model and key material parameters describing the material's performance are estimated. The ability to obtain such parameters facilitates material optimisation for a given application.
\end{abstract}

Keywords: Holography, holographic recording material, photopolymer, inhibition.

Contact Author: mgleeson@cs.nuim.ie and john.sheridan@ucd.ie

\section{INTRODUCTION}

Photopolymer materials and the photochemical kinetics associated with them [1-10] have been studied extensively in both academia and industry due to the growing interest in applications involving photopolymers [11-18]. In order to maximise the potential of these materials for various applications, the necessity for a physically comprehensive theoretical model of the effects which occur during photo-polymerization is becoming ever more important, [4,6-10,1928]. Providing such a model will enable potential trends in a material's performance to be recognized and optimised, $[19,29]$. Such models allow simulations of ratios of various key material components to be made, yielding indications of the most suitable material compositions in order to improve material performance.

In this paper we extend some recently published results on the Non-local Photo-polymerization Driven Diffusion (NPDD) model, $[9,10]$ in particular allowing for spatially and temporally varying primary radical generation. We then apply the improved model to analyse a number of effects observed to take place during holographic grating formation in an acrylamide/polyvinylalcohol (AA/PVA) based photopolymer which has been sensitised with a xanthene type dye, Erythrosin B [5], and compare experimental results and the predictions of the model with the aim of characterising these effects. 
The paper is structured as follows. In Section 2 we briefly examine the photochemical processes involved during holographic grating formation, reviewing some of the assumptions previously made. We then implement a more accurate representation of the initiation mechanisms occurring during exposure, thus increasing the physicality of the model proposed. The governing set of truncated first-order coupled differential equations can then be generated. Section 3 is split into two main sections, low intensity and high intensity recordings. By applying suitable initial conditions, the differential equations presented in Section 2 are solved numerically and simulations highlighting the predictions of the extended model are made for both low and high intensities. Growth curves of refractive index modulation are recorded. The low recording intensities emphasize the effects of inhibition, which are most clearly observed at the start of grating formation. The high recording intensities illustrate the effects of primary radical termination which is more dominant when large concentrations of primary radicals are present in the material. The model is then fit to the experimental data in order to extract estimates of key material parameters.

\section{PHOTOCHEMICAL PROCESSES}

\subsection{Review of kinetic models}

The photochemical processes, which are present during photo-polymerization, are complex [1-5,8,9,21,22], however an understanding of these processes is of utmost importance if a practical model is to be developed. In a recent review, [29] many of the assumptions made in developing photochemical models of free radical photo-polymerisation were discussed, [1-8,19-28]. A number of physical effects not included in the current models were listed, which indicated a lack of physicality under certain exposure conditions. Following the appearance of this review, a series of papers were published $[9,10]$ which addressed many of these issues and provided a model containing a consistent set of chemical reaction equations to take into account many of these effects. These effects included;

i) Removal of the steady state approximation for macroradical concentration,

ii) inclusion of spatially and temporally non-local polymer chain growth,

iii) inclusion of time varying photon absorption,

iv) simultaneously including the effects of both primary, i.e. $R^{\bullet}-M^{\bullet}$, and bimolecular, i.e. $M^{\bullet}-M^{\bullet}$, termination,

v) inclusion of the changes in the polymerization kinetic constants caused by increased viscosity, and finally

vi) inclusion of polymerization inhibiting effects.

The resulting Non-local Photo-polymerisation Driven Diffusion (NPDD) model was then experimentally verified by applying it to study (a) normalised transmission curves, and (b) growth curves of refractive index modulation for both short and continuous holographic exposures, in two significantly different free radical photopolymer materials [5,7,30]. The quality of the fits obtained to both photopolymer materials, indicated the versatility and applicability of the model.

In the past number of years, extensive work has been presented in the literature to describe the time varying absorption effects, which occur in photopolymer materials during exposure, [31-36]. In all cases the aim has been to improve the understanding of the photo-kinetics occurring in these materials, and critically to enable accurate predictions of the generation of primary radicals. A model of photosensitiser behaviour proposed by Carretero et al. [35], has recently been extended to account for: (i) photon absorption, (ii) the regeneration or recovery of absorptive photosensitiser, and (iii) photosensitiser bleaching, [31-34]. Using this model an expression for the time varying absorbed intensity, $I_{\mathrm{a}}(t)$ (Einstein $/ \mathrm{cm}^{3} \mathrm{~s}$ ), was derived and the values of key material parameters were estimated using non-linear fits of the dye model to experimentally obtained transmission curves. The processes of primary radical generation were then described using the simple expression,

$$
R_{i}=2 \Phi I_{a}(t)
$$
where $R_{\mathrm{i}}$ is the rate of generation of primary radicals, $\Phi$ is the number of primary radicals initiated per photon absorbed. The factor of two indicates that radicals are created in pairs, [9,10,31-37].

In this paper, we extend the NPDD model in $[9,10]$ by more accurately representing the temporal and spatial variation of photosensitiser concentration and the associated temporal and spatial generation and removal of primary radicals. As a result the number of approximations made in modelling the photo-initiation kinetics are significantly reduced. Thus a more physically accurate representation of the photo-polymerization kinetics is produced. Crucially, the increased 
physicality of the proposed model enables a more accurate analysis of the process of inhibition and primary radical termination.

\subsection{Reaction Mechanisms}

The kinetic model presented in this analysis is based upon the following four reaction processes.

I. Initiation,

$$
\begin{gathered}
I \stackrel{h v}{\longrightarrow} R^{\bullet}, \\
R^{\bullet}+M \stackrel{k_{i}}{\longrightarrow} M_{1}^{\bullet}=\text { Chain Initiator } .
\end{gathered}
$$

II. Propagation,

$$
M_{n}^{\bullet}+M \stackrel{k_{p}}{\longrightarrow} M_{n+1}^{\bullet}=\text { Growing Polymer Chain }
$$

III. Termination,

$$
\begin{gathered}
M_{n}^{\bullet}+M_{m}^{\bullet} \stackrel{k_{t c}}{\longrightarrow} M_{n+m}=\text { Dead Polymer } \\
M_{n}^{\bullet}+M_{m}^{\bullet} \stackrel{k_{t d}}{\longrightarrow} M_{n}+M_{m}=\text { Dead Polymer } \\
M_{n}^{\bullet}+R^{\bullet} \stackrel{k_{t p}}{\longrightarrow} M_{n} R=\text { Dead Polymer }
\end{gathered}
$$

IV. Inhibition,

$$
\begin{gathered}
D^{*}+Z \stackrel{k_{z, \text { Dye }}{ }^{*}}{\longrightarrow} \text { LeucoDye }+Z^{*}, \\
R^{\bullet}+Z \stackrel{k_{z, R^{\bullet}}}{\longrightarrow}\left(R+Z^{\bullet} \text {, and/or } R Z^{\bullet}\right)=\text { Scavenged Radical }, \\
M_{n}^{\bullet}+Z \stackrel{k_{z, M^{\bullet}}}{\longrightarrow}\left(M_{n}+Z^{\bullet} \text {, and/or } M_{n} Z^{\bullet}\right)=\text { Dead Polymer } .
\end{gathered}
$$

In the above set of chemical equations, $I$ is the initiator concentration, $h v$ indicates the energy absorbed from a photon, $M$ is the monomer concentration, $Z$ is the inhibitor concentration, $M_{n}, M_{m}, M_{n+m}, M_{n} R$ and $M_{n} Z^{\bullet}$ represent polymer species with no active propagating tip, i.e. Dead Polymer. $D^{*}$ is the concentration of excited photosensitiser and $Z^{*}$ is the concentration of singlet oxygen [33,34,37-39]. The term Dead Polymer signifies the cessation of the growth of a propagating macroradical of $n$ monomer repeat units, [37], while the term Scavenged Radical signifies the removal of a primary radical, [37-39]. $k_{\mathrm{p}}, k_{\mathrm{tc}}, k_{\mathrm{td}}, k_{z, M}$, and $k_{z, R^{\bullet}}\left(\mathrm{cm}^{3} \mathrm{~mol}^{-1} \mathrm{~s}^{-1}\right)$ are the rate constants of propagation, termination by combination, termination by disproportionation, inhibition of macroradicals and inhibition of primary radicals respectively.

\subsection{Primary Radical Production}

As can be seen in Eq (2), the initiation process involves two steps: The first step is the production of free radicals by homolytic dissociation of the initiator to yield an initiator (primary) radical, $R^{\bullet}$, i.e., Eq (2a). The second step is chain initiation, i.e., Eq (2b), in which the primary radicals produced due to the absorption of photons react with the monomer to produce the chain initiating species $M_{1}^{\bullet}$, [1-4,37]. The kinetic rate constant for this step is $k_{\mathrm{i}}\left(\mathrm{cm}^{3} \mathrm{~mol}^{-1} \mathrm{~s}^{-1}\right)$, i.e. the chain initiation kinetic constant. As stated the main extensions to the previous model $[9,10]$ involve improvements to the modelling of the temporal and spatial variations in primary radical production. Therefore, the main focus of this subsection will be the first step of the initiation mechanism, which is presented in Eq (2a). 
In order to do this, we assume that the following photochemical reactions, take place upon illumination of a photopolymer layer sensitised with a xanthene or thiazine type photosensitiser [5], with an appropriate wavelength. These are as follows,

$$
\begin{gathered}
D+h v \stackrel{k a}{\longrightarrow} D^{*}, \\
D^{*}+Z \stackrel{k z, D}{\longrightarrow} \text { Leuco Dye }, \\
D^{*} \stackrel{k r}{\longrightarrow} D, \\
C I+{ }^{*}+R^{\bullet}+H^{+}+D^{\bullet-} \longrightarrow R^{\bullet}+H D^{\bullet}, \\
C H{ }_{2} D+C I_{\text {int }},
\end{gathered}
$$

In these equations $D$ represents the concentration of photosensitiser (dye), $h v$ represents the photon energy incident on the material, $D^{*}$ is the excited state of the dye, $C I$ is the co-initiator, $R^{\bullet}$ represented the primary radical concentration, $Z$ is the inhibitor, $H D^{\bullet}$ represents a radicalised dye, which has abstracted a hydrogen from the co-initiator, and $H_{2} D$ is the di-hydro transparent form of the dye. $C I_{\text {int }}$ is an intermediate form of the co-initiator, which is no longer available for reaction. $k_{\mathrm{a}}\left(\mathrm{s}^{-1}\right)$ is the rate of production of excited state photosensitiser, $k_{\mathrm{r}}\left(\mathrm{s}^{-1}\right)$ is the rate of recovery or regeneration of photo-absorber, $k_{\mathrm{d}}\left(\mathrm{cm}^{3} \mathrm{~mol}^{-1} \mathrm{~s}^{-1}\right)$ is the rate of dissociation of the initiator and $k_{\mathrm{z}, \mathrm{D}}\left(\mathrm{cm}^{3} \mathrm{~mol}^{-1} \mathrm{~s}^{-1}\right)$ is the inhibition rate constant associated with the reaction with excited dye molecules. We note that previous models of the photo-initiation kinetics have not included all the reactions specified in Eq (6).

In order to use the proposed rate equations in the next subsection, it is first necessary to convert the exposure intensity $I_{0}$ $\left(\mathrm{mW} / \mathrm{cm}^{2}\right)$ to the appropriate units $\left(\right.$ Einsteins $\left./ \mathrm{cm}^{3} \mathrm{~s}\right)$. This can be done as follows, $I_{0}{ }^{\prime}=\frac{T_{s f} B I_{0}}{d}\left(\frac{\lambda}{N_{a} h c}\right)$, where $\lambda$ (nm) is the wavelength of incident light, $N_{\mathrm{a}}\left(\mathrm{mol}^{-1}\right)$ is Avogadro's constant, $c(\mathrm{~m} / \mathrm{s})$ is the speed of light, and $h(\mathrm{Js})$ is Plank's constant. $B=1-e^{-\varepsilon D_{0} d}$, is the absorptive fraction which determines a material layer's initial absorptive capacity and is a function of the dye's initial concentration, $T_{\mathrm{sf}}$ is a fraction associated with the light lost by Fresnel and scattering losses, [8,31-35] $D_{0}\left(\mathrm{~mol} / \mathrm{cm}^{3}\right)$, molar absorptivity, $\varepsilon\left(\mathrm{cm}^{2} / \mathrm{mol}\right)$ and material layer thickness, $d(\mathrm{~cm})$. The rate of production of the excited state photosensitiser, appearing in Eq (6.1) can then be represented by $k_{a}=\phi \varepsilon d I_{0}^{\prime}\left(\mathrm{s}^{-1}\right)$, where $\phi$ (mol/Einstein) is the quantum efficiency of the reaction [37]. Therefore, if the photosensitiser's initial concentration, molar absorptivity, quantum efficiency, and layer thickness are known, the rate of generation of excited state photosensitiser, $D^{*}$, can be determined for a given exposure intensity.

\subsection{Model Development}

In the case of holographic illumination, there is a spatial distribution of irradiance, which in our case is typically cosinusoidal. In this case the incident intensity is represented as $I(x, t)=I_{0}^{\prime}[1+V \cos (K x)]$, where $V$ is the fringe visibility and $K=2 \pi / \Lambda$, where $\Lambda$ is the grating period. The mechanisms, which are presented in Eq (6), can then be represented by a set of coupled differential equations. The combination of these equations is equivalent to the previous representation of primary radical production in time and space, which is presented in Eq (1). Combining these coupled 
differential equations with those previously presented in Ref $[9,10]$ for the mechanisms of initiation, propagation, termination and inhibition, yields the following set of first-order coupled differential equations governing the photosensitiser:

$$
\begin{gathered}
\frac{d D(x, t)}{d t}=-k_{a} D(x, t)+k_{r} D^{*}(x, t), \\
\frac{d D^{*}(x, t)}{d t}=k_{a} D(x, t)-k_{r} D^{*}(x, t)-k_{d} D^{*}(x, t) C I(x, t)-k_{z, D} D^{*}(x, t) Z(x, t), \\
\frac{d C I(x, t)}{d t}=-k_{d} D^{*}(x, t) C I(x, t)-k_{b} H D^{\bullet}(x, t) C I(x, t), \\
\frac{d H D^{\bullet}(x, t)}{d t}=k_{d} D^{*}(x, t) C I(x, t)-k_{b} H D^{\bullet}(x, t) C I(x, t),
\end{gathered}
$$

As in the previous analysis, $[9,10]$ it is assumed that the effect of inhibition during exposure is due solely to the initially dissolved oxygen present within the photopolymer layer. The non-uniform recording irradiance causes concentration gradients of oxygen as it is consumed in inhibitory reactions. This then results in the diffusion of oxygen from the dark non-illuminated regions to the bright illuminated regions. As oxygen molecules are small compared to the other material components which constitute the photopolymer layer, it can be assumed that the oxygen is relatively free to diffuse rapidly, resulting in a one-dimensional standard diffusion equation for the concentration of inhibitor,

$$
\begin{aligned}
\frac{d Z(x, t)}{d t}=\frac{d}{d x}\left[D_{z} \frac{d Z(x, t)}{d x}\right]- & k_{z, D} D^{*}(x, t) Z(x, t) \\
& -k_{z, R^{*}} Z(x, t) R^{\bullet}(x, t)-k_{z, M} . Z(x, t) M^{\bullet}(x, t),
\end{aligned}
$$

where $Z$ is the instantaneous inhibiting oxygen concentration and $D_{z}$ is the diffusion constant of oxygen in the dry material layer, which in this analysis will be assumed to be time and space independent. This assumption is reasonable, as this fast rate of diffusion of the small oxygen molecule will not be significantly affected by any small changes in material viscosity. The inhibition rate constants, $k_{z, R^{*}}$ and $k_{z, M^{*}}$, will in general have different values (of reactivity) due to the differences in the relative molecular size, [37]. However in this analysis, for the sake of simplicity we assume $k_{z}=k_{z, R^{\bullet}}=k_{z, M}$. Furthermore it is expected that the reactivity of oxygen with the excited state form of the photosensitiser will be much lower, i.e. $k_{\mathrm{z}, \mathrm{D}}<<k_{\mathrm{z}}$ and therefore we assume it is negligible in this analysis, [33]. As before $[9,10]$, it is assumed that the inhibition rate constant can be expressed as,

$$
k_{z}=k_{z, 0} \exp \left(-E_{z} / R T\right),
$$

where in this equation $k_{\mathrm{z}, 0}\left(\mathrm{~cm}^{3} \mathrm{~mol}^{-1} \mathrm{~s}^{-1}\right)$ is the Arrhenius pre-exponential factor, $E_{\mathrm{z}}=18.23 \times 10^{3}\left(\mathrm{Jmol}^{-1}\right)$ is the activation energy of oxygen, (i.e., the energy that must be overcome in order for oxygen to react with the given species), $R=8.31$ $\left(\mathrm{JK}^{-1} \mathrm{~mol}^{-1}\right)$ is the universal gas constant, and $T(\mathrm{~K})$ is the local temperature [37].

The equation governing the concentration of primary radicals, including the new term for primary radical generation, is given by

$$
\frac{d R^{\bullet}(x, t)}{d t}=k_{d} D^{*}(x, t) C I(x, t)-k_{i} R^{\bullet}(x, t) u(x, t)-k_{t p} R^{\bullet}(x, t) M^{\bullet}(x, t)-k_{z} R^{\bullet}(x, t) Z(x, t),
$$


where $u(x, t)$ is the free-monomer concentration, (denoted earlier in the chemical reactions by $M$ ). This equation states that the rate of change of primary radical concentration is proportional to the concentration of primary radicals generated by photon absorption, minus the amounts removed by: (a) the initiation of macroradicals, (b) primary termination with growing polymer chains, and (c) inhibition by oxygen.

Including both types of termination mechanism (primary and bimolecular) and the effects of inhibition, the equation governing macroradical concentration is then

$$
\frac{d M^{\bullet}(x, t)}{d t}=k_{i} R^{\bullet}(x, t) u(x, t)-2 k_{t}\left[M^{\bullet}(x, t)\right]^{2}-k_{t p} R^{\bullet}(x, t) M^{\bullet}(x, t)-k_{z} Z(x, t) M^{\bullet}(x, t),
$$

where the squared term represents the effects of bimolecular termination. The generation term in this equation previously appears as the removal term due to macroradical initiation in Eq (13).

The non-uniform irradiance creates monomer concentration gradients, and as a result monomer diffuses from the dark regions to the monomer depleted exposed regions. This results in a spatial polymer concentration distribution, which provides the modulation of refractive index in the material, i.e., the holographic grating. We represent the monomer concentration using the following $1 \mathrm{D}$ diffusion equation,

$$
\frac{d u(x, t)}{d t}=\frac{d}{d x}\left[D_{m}(x, t) \frac{d u(x, t)}{d x}\right]-k_{i} R^{\bullet}(x, t) u(x, t)-\int_{-\infty}^{\infty} k_{p} M^{\bullet}\left(x^{\prime}, t\right) u\left(x^{\prime}, t\right) G\left(x, x^{\prime}\right) d x^{\prime},
$$

where $D_{\mathrm{m}}(x, t)$ represents the monomer diffusion constant. $G\left(x, x^{\prime}\right)$ is the non-local material spatial response function given by:

$$
G\left(x, x^{\prime}\right)=\frac{1}{\sqrt{2 \pi \sigma}} \exp \left[\frac{-\left(x-x^{\prime}\right)^{2}}{2 \sigma}\right]
$$

where $\sigma$ is the constant non-local response parameter normalized with respect to the grating period, $\Lambda$. This non-local spatial response function represents the effect of initiation at location $x^{\prime}$ on the amount of monomer polymerized at location $x$.

The equation governing the polymer concentration is

$$
\frac{d N(x, t)}{d t}=\int_{-\infty}^{\infty} k_{p} M^{\bullet}\left(x^{\prime}, t\right) u\left(x^{\prime}, t\right) G\left(x, x^{\prime}\right) d x^{\prime}-\frac{d}{d x}\left[D_{N}(x, t) \frac{d N(x, t)}{d x}\right],
$$

where $D_{\mathrm{N}}(x, t)$ represents the polymer diffusion constant. As with the monomer above in Eq (15), the non-uniform irradiance creates a polymer concentration distribution. If the polymer chains are not cross-linked sufficiently, they will tend to diffuse out of the exposed regions in order to reduce the polymer gradient, [8]. If this takes place it will result in a decay of the grating strength with time. However in this paper, we assume there is sufficient cross-linking and that $D_{\mathrm{N}}(x, t)=0$, i.e., we record very stable gratings, as seen in the analysis presented in Ref [8], which uses the same material composition.

Since all the above equations presented in Eqs (7-11), (13-15) and (17), depend upon the spatial distribution of the exposing intensity, they will all be periodic even functions of $x$ and can therefore be written as Fourier series, i.e., $X(x, t)=\sum_{j=0}^{\infty} X_{j}(t) \cos (j K x)$, where $X$ represents the species concentrations, $D, D^{*}, C I, H D^{\bullet}, R^{\bullet}, M^{\bullet}, u, N$ and $Z$.

A set of first-order coupled differential equations can then be obtained in the same manner presented in Refs [9,10], by gathering the coefficients of the various co-sinusoidal spatial contributions and writing the equations in terms of these time varying spatial harmonic amplitudes. These coupled equations can then be solved using the following initial conditions, 


$$
\begin{gathered}
Z_{0}(t=0)=Z_{0}, D_{0}(t=0)=D_{0}, C I_{0}(t=0)=C I_{0}, u_{0}(t=0)=U_{0}, \\
D_{n>0}(t=0)=D_{n \geq 0}^{*}(t=0)=H D_{n \geq 0}^{\cdot}(t=0)=C I_{n>0}(t=0)=0, \text { and } \\
Z_{n>0}(t=0)=R_{n \geq 0}^{\bullet}(t=0)=M_{n \geq 0}^{\bullet}(t=0)=N_{n \geq 0}(t=0)=0 .
\end{gathered}
$$

As in previous analysis the Fourier series expansion of the monomer and polymer harmonics involves use of the nonlocal response parameter $G\left(x, x^{\prime}\right)$ which is represented in the coupled differential equations by $S_{i}=\exp \left(-i^{2} K^{2} \sigma / 2\right)$.

\section{THEORETICAL AND EXPERIMENTAL RESULTS}

Before applying the theoretical model presented in the previous section to experimental data we wish to examine its general behaviour. In all theoretical simulations presented here, it is assumed that time varying viscosity effects are negligible and therefore, $D_{\mathrm{m}}(x, t)=D_{\mathrm{m} 0}=8.0 \times 10^{-11} \mathrm{~cm}^{2} / \mathrm{s}$, [22]. All kinetic parameter values are assigned appropriate values, which are typical for the AA/PVA photopolymer material examined here, $[5,7,9,10]$.

\subsection{Low Intensities}

12 spatial concentration harmonics are retained in the simulations, solved using the initial conditions presented in Eq (18) with $U_{0}=2.83 \times 10^{-3} \mathrm{~mol} / \mathrm{cm}^{3}, D_{0}=1.22 \times 10^{-6} \mathrm{~mol} / \mathrm{cm}^{3}, C I_{0}=3.18 \times 10^{-3} \mathrm{~mol} / \mathrm{cm}^{3}$ and $Z_{0}=1 \times 10^{-8} \mathrm{~mol} / \mathrm{cm}^{3}$. Assuming typical recording conditions for an unslanted transmission type volume holographic grating, for $\Lambda=700 \mathrm{~nm}$ and fringe visibility $V=1$, simulations of the temporal and spatial variation in the photosensitiser concentration, $D(x, t)$, are generated and presented in Figure 1.

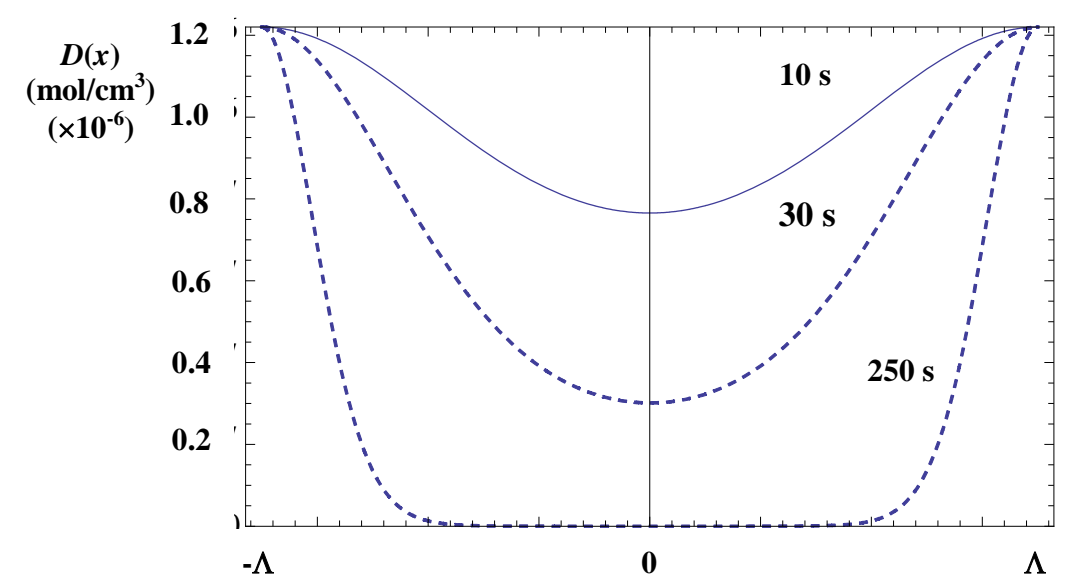

Figure 1. Simulation of the spatial variation of the ground state photosensitiser concentration for an exposure intensity $I_{0}=$ $1 \mathrm{~mW} / \mathrm{cm}^{2}$, at $\Lambda=700 \mathrm{~nm}$, for various exposure times, $t_{\exp }=10$ s (joined line), $t_{\exp }=30$ s (small dashed line), $t_{\exp }=250 \mathrm{~s}$ (dashed line).

The typical rate constants used were $k_{\mathrm{p}}=k_{\mathrm{i}}=2.65 \times 10^{7} \mathrm{~cm}^{3} / \mathrm{mol} \mathrm{s}, k_{\mathrm{t}}=6 \times 10^{8} \mathrm{~cm}^{3} / \mathrm{mol} \mathrm{s}, k_{\mathrm{tp}}=k_{\mathrm{t}} \times 10, k_{\mathrm{d}}=k_{\mathrm{b}}=1.6 \times 10^{3}$ $\mathrm{cm}^{3} / \mathrm{mol} \mathrm{s}, k_{\mathrm{z}}=3 \times 10^{12} \mathrm{~cm}^{3} / \mathrm{mol} \mathrm{s}$ and $k_{\mathrm{r}}=1.22 \times 10^{-3} \mathrm{~s}^{-1}$, [9,10]. For an exposure intensity of $I_{0}=1 \mathrm{~mW} / \mathrm{cm}^{2}$ and $\lambda=532$ $\mathrm{nm}$, the absorption parameters estimated from fits to normalised transmission curves for a material layer of thickness $d$ $=100 \mu \mathrm{m}$ were, $\varepsilon=1.4 \times 10^{8} \mathrm{~cm}^{2} / \mathrm{mol}, \phi=0.066 \mathrm{~mol} /$ Einstein and $T_{\mathrm{sf}}=0.76$, with $N_{\mathrm{a}}=6.02 \times 10^{23} \mathrm{~mol}^{-1}, c=3 \times 10^{8} \mathrm{~ms}^{-1}$ and $h=6.62 \times 10^{-34} \mathrm{Js}$, [31-34]. The oxygen diffusion coefficient was assumed to be $D_{\mathrm{z}}=1.0 \times 10^{-8} \mathrm{~cm}^{2} / \mathrm{s}$. The parameter $S_{1}$, which quantifies the extent of the non-locality in the first harmonic coupled differential equation, was chosen to have a value of $S_{1}=0.94$. This corresponds to a non-local response length of $\sqrt{\sigma^{\prime}}=54 \mathrm{~nm}$, [8]. 
As can be observed from Figure 1, the spatial sinusoidal variation in the exposing interference pattern causes a rapid consumption of the ground state dye in the bright illuminated regions. As the exposure time increases the sinusoidal variation of the dye concentration is distorted and the width of the non-illuminated dark bands narrows. This loss in sinusoidal fidelity results in a spatial production of primary radicals, which deviates significantly from the sinusoidal primary radical generation term presented in Eq (1). Subsequently, this yields a non-linear material response, as the number of polymer chains initiated, (discussed in Section 2), are not simply generated in direct proportion to the exposing interference pattern. This is an important prediction of the model, which agrees well with experimental observation.

Figure 2, shows a simulation of growth curves of refractive index modulation with varying values of the concentration of initially dissolved oxygen, $Z_{0}\left(\mathrm{~mol} / \mathrm{cm}^{3}\right)$, under the same conditions as the previous figures but with $Z_{0}=1 \times 10^{-8}$ $\mathrm{mol} / \mathrm{cm}^{3}$ (joined line), $Z_{0}=5 \times 10^{-8} \mathrm{~mol} / \mathrm{cm}^{3}$ (short dashed line), and $Z_{0}=1 \times 10^{-7} \mathrm{~mol} / \mathrm{cm}^{3}$ (long dashed line). As the concentration of inhibitor is increased, the inhibition time, $t_{\mathrm{i}}$ increases as expected, i.e., more inhibitor causes a greater scavenging of the primary- and macro-radicals. Comparing the experimental results with the theoretical prediction, it became clear that when using the model as presented, the trend of increased inhibition times, $t_{\mathrm{i}}$, for reduced exposure intensities, did not satisfactorily replicate the experimental behavior observed. In order to achieve good fits to the experimental data, it was found necessary to increase the initial concentration of dissolved oxygen available in the photopolymer layer, $Z_{0}$, as the recording intensities were reduced. The variation between the experimental observation and theoretical prediction was as much as $8 \mathrm{~s}$ for the lowest recording intensity examined in this paper for unsealed layers. This divergence between experiment and prediction suggests that the model is incomplete and, that in order to mimic this physically observed behavior, amendments to the model are necessary.

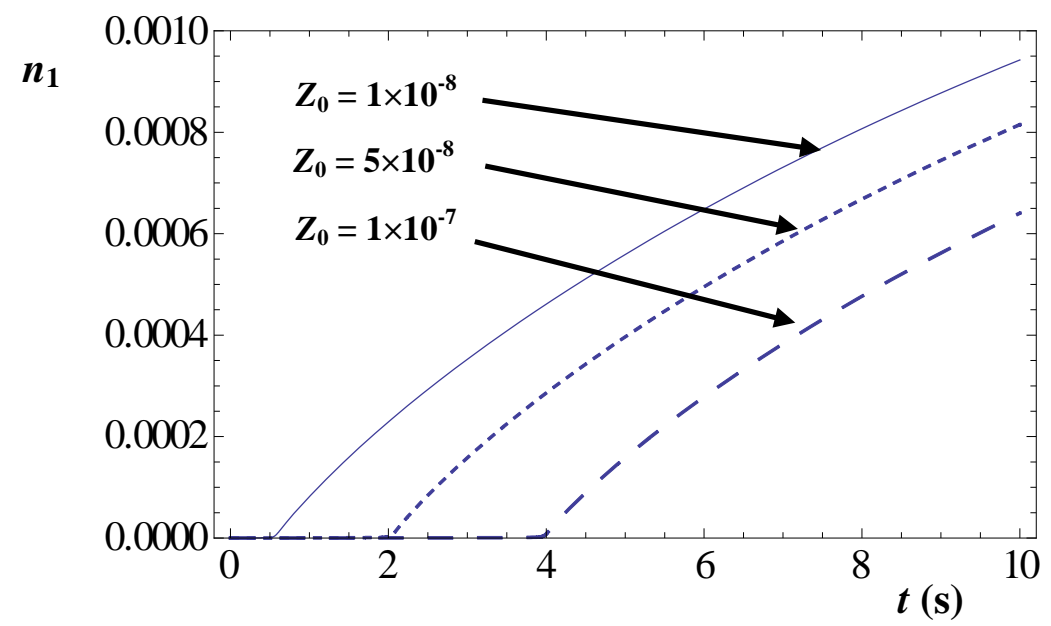

Figure 2. Simulations of refractive index modulation with time, for various values of dissolved oxygen concentration. $Z_{0}=1 \times 10^{-7} \mathrm{~mol} / \mathrm{cm}^{3}$ (long dashed line), $Z_{0}=5 \times 10^{-8} \mathrm{~mol} / \mathrm{cm}^{3}$ (short dashed line) and $Z_{0}=1 \times 10^{-8} \mathrm{~mol} / \mathrm{cm}^{3}$ (joined line).

In a previous paper [38], it was found that by cover-plating or sealing the photopolymer layer with glass slides, the inhibition times observed during exposure compared with the uncoverplated or unsealed layers were significantly reduced. These effects were attributed to the removal or reduction of oxygen diffusing in from the surrounding environment, which was replacing or replenishing the oxygen consumed during exposure. It must be noted at this point, that the experimental data examined here were uncoverplated photopolymer layers, which were subject to this external oxygen diffusion.

In order to represent this process in the model, an additive term representing the replenishing of inhibiting oxygen from the outside surrounding air, into the material layer, was included. Therefore, Eq (11) must be revised and becomes 


$$
\begin{aligned}
\frac{d Z(x, t)}{d t}=\frac{d}{d x}\left[D_{z} \frac{d Z(x, t)}{d x}\right]-k_{z, D} D^{*}(x, t) Z(x, t)-k_{z, R^{*}} Z(x, t) R^{\bullet}(x, t) & \\
& -k_{z, M} \cdot Z(x, t) M^{\bullet}(x, t)+\tau_{z}\left[Z_{0}-Z(x, t)\right],
\end{aligned}
$$

where $\tau_{\mathrm{z}}$ represents the rate of replenishing of oxygen into the material layer. We note that it is assumed that the oxygen concentration can never be larger than the original dissolved oxygen concentration, $Z_{0}\left(\mathrm{~mol} / \mathrm{cm}^{3}\right)$ and that this additive term is assumed to be constant in space. In order to illustrate these effects Figure 3 shows a simulation of the behavior of the oxygen concentration with varying values of the replenishing constant, $\tau_{\mathrm{z}}$, for an exposure intensity, $I_{0}=$ $0.04 \mathrm{~mW} / \mathrm{cm}^{2}$ and exposure time, $t_{\exp }=30 \mathrm{~s}$. As can be observed, an increase in $\tau_{\mathrm{z}}$ results in: an increase of the inhibition period, and an increase in the rate at which oxygen returns to its original dissolved oxygen concentration.

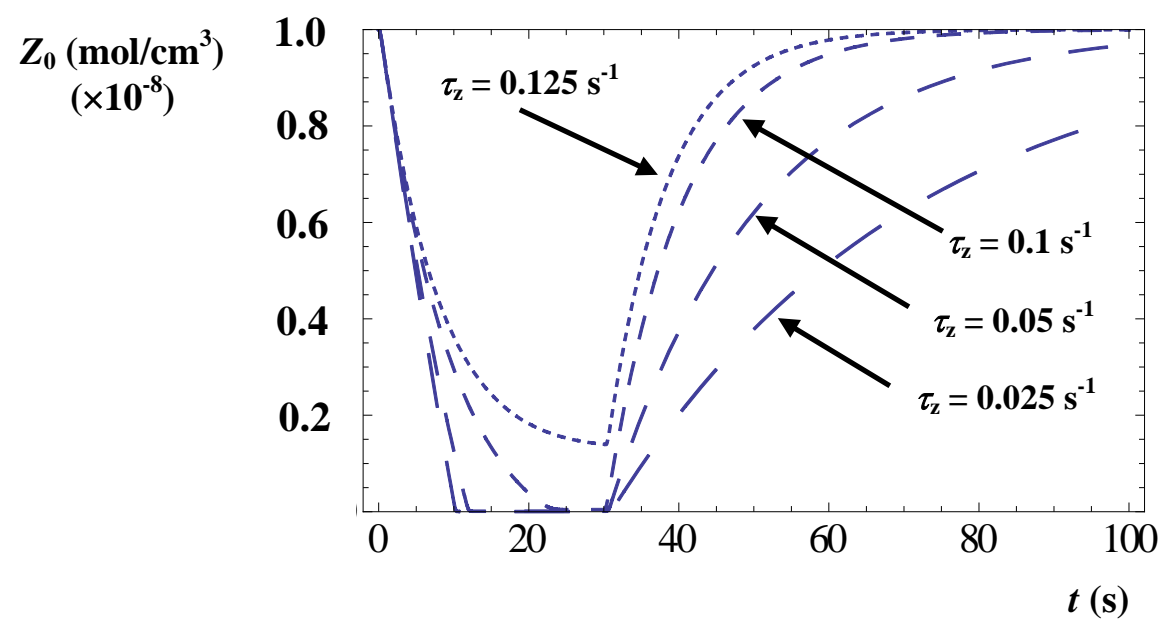

Figure 3. Behaviour of the oxygen concentration with varying values of $\tau_{\mathrm{z}}$, for an exposure time, $t_{\text {exp }}=30 \mathrm{~s}$ and exposure intensity, $I_{0}$ $=0.04 \mathrm{~mW} / \mathrm{cm}^{2} . \tau_{\mathrm{z}}=0.125 \mathrm{~s}^{-1}$ (shortest dash), $\tau_{\mathrm{z}}=0.1 \mathrm{~s}^{-1}$ (short dash), $\tau_{\mathrm{z}}=0.05 \mathrm{~s}^{-1}$ (long dash), $\tau_{\mathrm{z}}=0.025 \mathrm{~s}^{-1}$ (longest dash).

Implementing the appropriate Fourier series expansion to Eq (21) under the same initial conditions, the model is then applied to the experimental growth curves recorded in uncoverplated layers, yielding much more accurate fits to the data. Figure 4 shows a subset of this data with the corresponding fits obtained using the model.

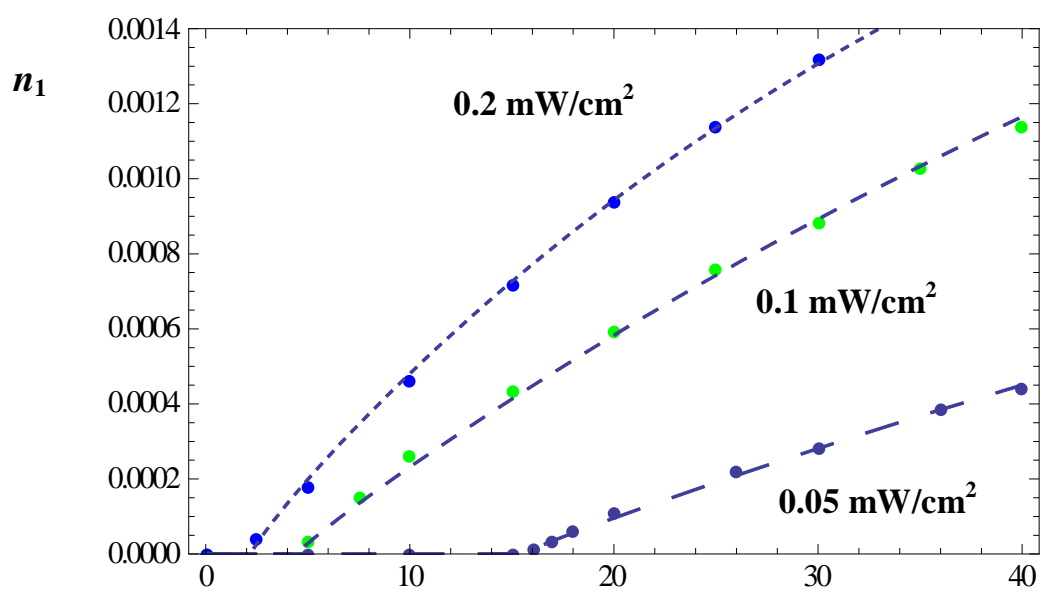

$t(s)$

Figure 4. Experimentally obtained growth curves of refractive index modulation recorded in uncoverplated AA/PVA photopolymer material layers at a spatial frequency of 1428 lines $/ \mathrm{mm}$ for 3 different exposing intensities, $I_{01}=0.2 \mathrm{~mW} / \mathrm{cm}^{2}$ (short dash), $I_{02}=0.1$ $\mathrm{mW} / \mathrm{cm}^{2}$ (dashed) and $I_{03}=0.05 \mathrm{~mW} / \mathrm{cm}^{2}$ (long dash) with corresponding fits achieved with the theoretical model. 
Some of the parameter values which were obtained from the fits to various intensities are, $k_{\mathrm{d}}=1.6 \times 10^{3} \mathrm{~cm}^{3} / \mathrm{mol} \mathrm{s}, k_{\mathrm{r}}=$ $1.2 \times 10^{-3} \mathrm{~s}^{-1}, k_{\mathrm{z}}=3.0 \times 10^{12} \mathrm{~cm}^{3} / \mathrm{mol} \mathrm{s}, D_{\mathrm{z}}=1 \times 10^{-8} \mathrm{~cm}^{2} / \mathrm{s}$, and it was assumed in all fits that $k_{\mathrm{tp}}=10 \times k_{\mathrm{t}} \mathrm{cm}^{3} / \mathrm{mol} \mathrm{s}$, and $k_{\mathrm{i}}=k_{\mathrm{p}} \mathrm{cm}^{3} / \mathrm{mol} \mathrm{s}$. The most significant values extracted from the fits are presented in Table 1 along with the parameter search ranges, which were used to obtain a best fit between experimental and theoretical prediction. These search ranges are typical of the valued presented in the literature for similar photopolymer materials, [3,4,37]. The Mean Squared Error (MSE) between the fit and the data are also included to indicate the quality of the fits.

\begin{tabular}{ccccccc}
\hline $\begin{array}{c}\mathbf{I}_{\mathbf{0}} \\
\left(\mathbf{m W} / \mathbf{c m}^{2}\right)\end{array}$ & $\begin{array}{c}\boldsymbol{t}_{\mathbf{i}} \\
(\mathbf{s})\end{array}$ & $\begin{array}{c}\boldsymbol{k}_{\mathbf{p}} \\
\left(\mathbf{c m}^{\mathbf{3}} / \mathbf{m o l s}\right) \\
\left(\times \mathbf{1 0}^{\mathbf{7}}\right)\end{array}$ & $\begin{array}{c}\boldsymbol{k}_{\mathbf{t}} \\
\left(\mathbf{c m}^{\mathbf{3}} / \mathbf{m o l s}\right) \\
\left(\times \mathbf{1 0}^{\mathbf{8}}\right)\end{array}$ & $\begin{array}{c}\boldsymbol{D}_{\mathbf{m 0}} \\
\left(\mathbf{c m}^{\mathbf{2}} / \mathbf{s}\right) \\
\left(\times \mathbf{1 0}^{-\mathbf{1 1}}\right)\end{array}$ & $\begin{array}{c}\tau_{\mathbf{z}} \\
\left(\mathbf{s}^{-1}\right)\end{array}$ & MSE \\
\hline $\mathbf{0 . 2 0}$ & 2.50 & 2.42 & 5.0 & 8.0 & 0.075 & 1.05 \\
$\mathbf{0 . 1 0}$ & 4.50 & 2.52 & 7.0 & 9.0 & 0.080 & 2.86 \\
$\mathbf{0 . 0 5}$ & 16.00 & 3.00 & 7.0 & 10.0 & 0.115 & 1.88 \\
\hline Search Range & - & $0.1-5.0$ & $0.1-9.0$ & $1.0-12.0$ & - & - \\
\hline
\end{tabular}

Table 1. Parameters extracted from fits to experimentally obtained growth curves of refractive index modulation in uncoverplated photopolymer layers.

As can be observed from Figure 4, the fit quality is very good and the model predicts the observed trend, that a reduction in the exposure intensity causes an increase in the inhibition period due to (i) initially dissolved oxygen and (ii) oxygen diffusion into the material from the surrounding air. It can also be seen that there is a reduction in the propagation and termination rates with increasing exposure intensities. This is most likely due to the increased viscosity effects, which occur due to increased conversion of monomer to polymer [2-4,9]. This is consistent with the results obtained from the previous model, [10]. It must also be noted at this point that the estimates obtained for the rates of propagation and termination are slightly higher than those reported in the previous published work by the authors, [10]. This is as a result of a more physically accurate description of the primary radical generation introduced by the model development. However, the estimated values extracted from the fits still remain well within the accepted ranges presented in the literature for similar photopolymer materials, [3,4,37].

In order to verify the necessity for the inclusion of the additive oxygen replenishing term in Eq (21), several growth curves of refractive index modulation were recorded in coverplated layers. These growth curves were recorded under the same conditions as the uncoverplated layers presented in Figure 4. Figure 5 shows experimental growth curves recorded at an exposure intensity of $I_{0}=0.05 \mathrm{~mW} / \mathrm{cm}^{2}$, in both coverplated and uncoverplated layers. The subsequent fits to the experimental data, which were achieved using the revised model are represented as short dash line (coverplated) and long dash line (uncoverplated).

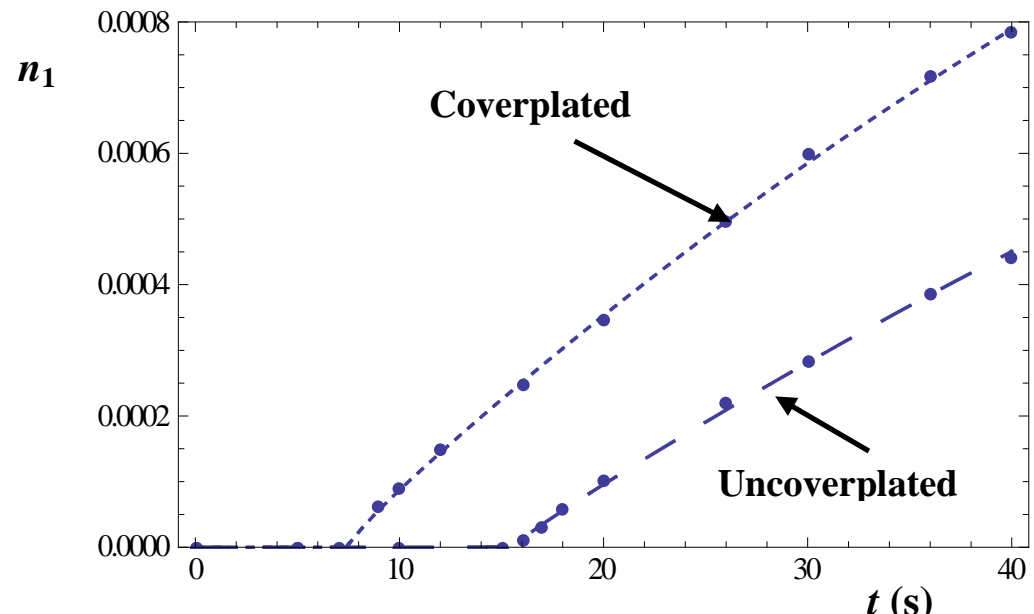

Figure 5. Experimentally obtained growth curves of refractive index modulation recorded $\stackrel{t}{\mathbf{t}}$ (S) both coverplated (short dash) and uncoverplated (long dash) AA/PVA photopolymer material layers at a spatial frequency of 1428 lines/mm for a recording intensity $I_{0}$ $=0.05 \mathrm{~mW} / \mathrm{cm}^{2}$ with corresponding fits achieved with the model. 
As can be observed from the figure there is a significant reduction in the inhibition period, from $t_{\mathrm{i}}=16 \mathrm{~s}$ (uncoverplated) to $t_{\mathrm{i}}=9 \mathrm{~s}$ (coverplated). As stated above, this is attributed to a reduction in the amount of oxygen available to diffuse into the layer from the surrounding air.

\begin{tabular}{ccccccc}
\hline & $\begin{array}{c}\mathbf{t}_{\mathbf{i}} \\
(\mathbf{s})\end{array}$ & $\begin{array}{c}\boldsymbol{k}_{\mathbf{p}} \\
\left(\mathbf{c m}^{\mathbf{3}} / \mathbf{m o l s}\right) \\
\left(\times \mathbf{1 0}^{\mathbf{7}}\right)\end{array}$ & $\begin{array}{c}\boldsymbol{k}_{\mathbf{t}} \\
\left(\mathbf{c m}^{3} / \mathbf{m o l s}\right) \\
\left(\times \mathbf{1 0}^{\mathbf{8}}\right)\end{array}$ & $\begin{array}{c}\boldsymbol{D}_{\mathbf{m} 0} \\
\left(\mathbf{c m}^{2} / \mathbf{s}\right) \\
\left(\times \mathbf{1 0}^{-11}\right)\end{array}$ & $\begin{array}{c}\tau_{\mathbf{z}} \\
\left(\mathbf{s}^{-1}\right)\end{array}$ & MSE \\
\hline Coverplated & 9.0 & 2.9 & 7.0 & 10.0 & 0.000 & 2.26 \\
Uncoverplated & 16.0 & 3.0 & 7.0 & 10.0 & 0.115 & 1.88 \\
\hline Search Range & - & $0.1-5.0$ & $0.1-9.0$ & $1.0-12.0$ & - & - \\
\hline
\end{tabular}

Table 2. Parameters extracted from fits to experimentally obtained growth curves recorded at $I_{0}=0.05 \mathrm{~mW} / \mathrm{cm}^{2}$ for a coverplated and uncoverplated polymer layer.

The estimated parameters extracted from these fits are presented in Table 2. The values determined for the replenishing rate $\tau_{\mathrm{z}}$, are consistent with what is experimental observed. In the case of the coverplated material layer, no oxygen can diffuse into the layer, i.e. $\tau_{\mathrm{z}}=0$.

\subsection{High Intensity}

In the previous section, we used low exposure intensities in order to highlight or emphasise the photopolymer material's inhibition behaviour. Similarly in this subsection we now examine the effects the materials response at high exposure intensities in order to illustrate and examine the behaviour of primary radical termination. All simulations are performed using the same parameter values as that presented in subsection 3.1 unless otherwise stated in the text.

We firstly model the behaviour of the material under normal illumination, i.e. no interference pattern. In this way, the fringe visibility is set to zero, i.e. $V=0$, resulting in a homogenous polymerisation of the exposed region of the material. The simulations generated represent the accumulative terminations caused by primary radicals (primary radical termination) for each of the recording intensities applied. We examine four specific cases for illustration in Figure 6 for a range of intensities from $10 \mathrm{~mW} / \mathrm{cm}^{2}$ up to $200 \mathrm{~mW} / \mathrm{cm}^{2}$. These are: (a) $k_{\mathrm{tp}}=k_{\mathrm{t}}=8 \times 10^{8} \mathrm{~cm}^{3} / \mathrm{mol} \mathrm{s}$, (b) $k_{\mathrm{tp}}=10 k_{\mathrm{t}}$, (c) $k_{\mathrm{tp}}=100 k_{\mathrm{t}}$ and (d) $k_{\mathrm{tp}}=1000 k_{\mathrm{t}}$. The exposure duration is suitably adjusted to ensure an equal dosage, $\xi=\sqrt{I_{0}} t$, is delivered to the material for each case examined. As can be easily observed from the figure, in going from cases (a) to (d), the overall macro-radical concentration terminated by primary radicals, $[P T]$, generally increases, for the increased values of $k_{\mathrm{tp}}$.

In cases (a) and (b), when the primary termination rate is comparable to the bimolecular termination rate, i.e., $k_{\mathrm{tp}}=k_{\mathrm{t}}$ and $k_{\mathrm{tp}}=10 k_{\mathrm{t}}$, the behaviour predicted is quite similar: the concentrations of macro-radicals terminated by primary termination decreases for the $I_{0}$ values from 10 to $60 \mathrm{~mW} / \mathrm{cm}^{2}$. Further increases in exposing intensity above 60 $\mathrm{mW} / \mathrm{cm}^{2}$ do not cause any further reduction. Examining cases (a) and (b), we see that $[P T]$ tends to decrease as the exposing intensity increases. This would suggest that more primary radicals are being used for initiation than for termination. For intensities higher than $60 \mathrm{~mW} / \mathrm{cm}^{2}$, $[P T]$ remains almost constant as the macro-radical concentration is exhausted .

However, in case (c) when $k_{\mathrm{tp}}=100 k_{\mathrm{t}}$, primary termination starts performing differently from cases (a) and (b), once the exposure intensity is greater than $60 \mathrm{~mW} / \mathrm{cm}^{2}$. As can be seen, the amount of $[P T]$ increases as the exposing intensity increases. This behaviour indicates that when $k_{\mathrm{tp}}$ is greater than $k_{\mathrm{t}}$ and the exposing intensity is significantly high, more growing macro-radicals will be involved in primary termination, as in Figure 6(c), before they continue propagating any longer or terminate one another by bimolecular termination. As demonstrated in case (d), the high intensity response of primary termination becomes even more significant than in case (c), when $k_{\mathrm{tp}}=1000 k_{\mathrm{t}}$. [PT] increases almost linearly as the exposure intensity increases, as shown in Figure 6(d). In this regime, primary radicals are much more available for termination, and are not limited by the available macro-radical concentration as in the scenarios predicted for cases (a) and (b). 


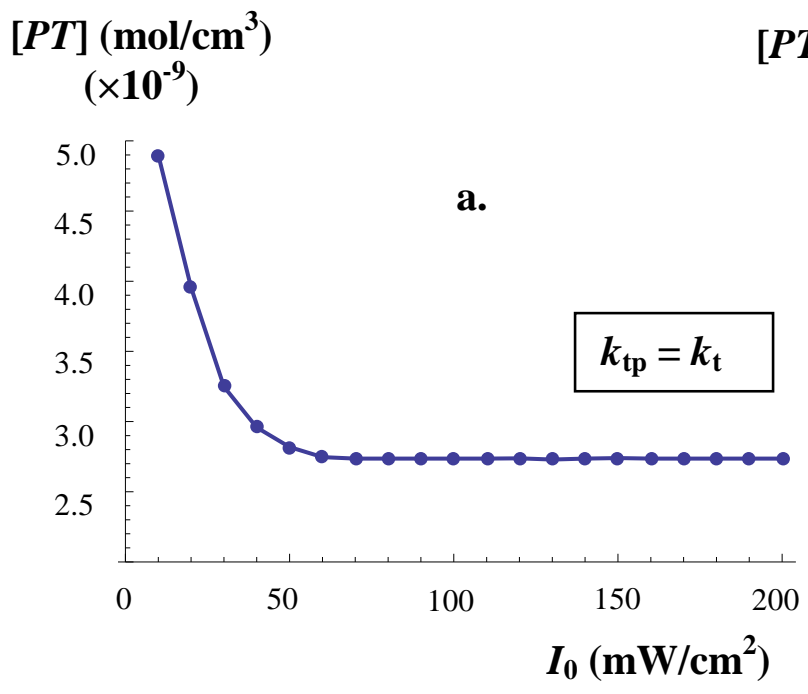

$[P T]\left(\mathrm{mol} / \mathrm{cm}^{3}\right)$

$\left(\times 10^{-8}\right)$

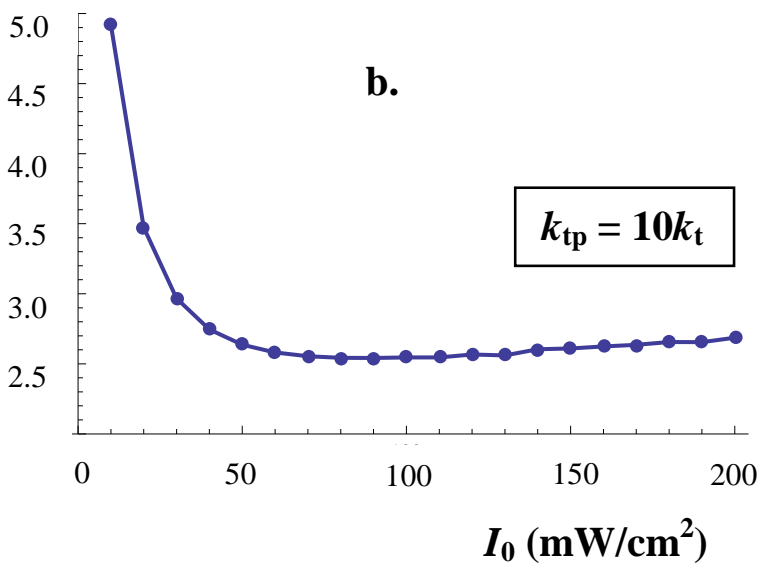

\section{$[P T]\left(\mathrm{mol} / \mathrm{cm}^{3}\right)$ \\ $\left(\times 10^{-7}\right)$}

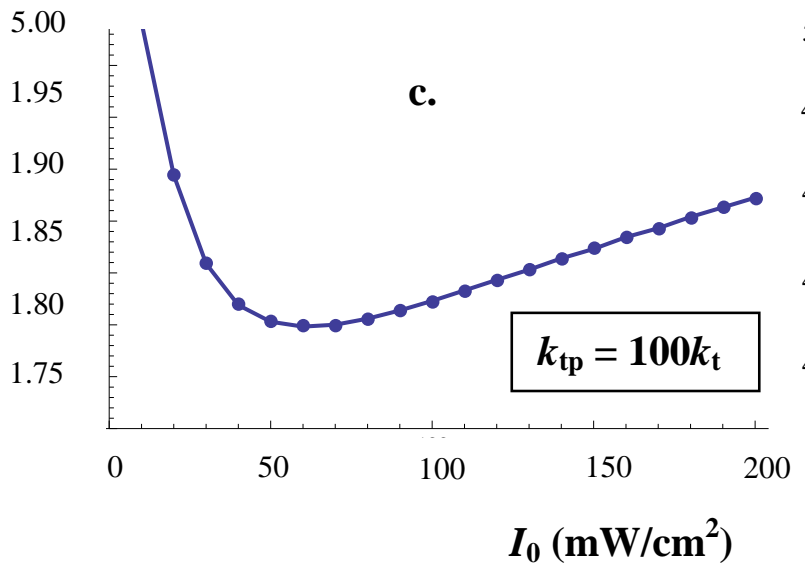

$[P T]\left(\mathbf{m o l} / \mathrm{cm}^{3}\right)$ $\left(\times 10^{-7}\right)$

Figure. 6. Intensity response of primary termination for cases: (a) $k_{\mathrm{tp}}=k_{\mathrm{t}}$, (b) $k_{\mathrm{tp}}=10 k_{\mathrm{t}}$, (c) $k_{\mathrm{tp}}=100 k_{\mathrm{t}}$ and (d) $k_{\mathrm{tp}}=1000 k_{\mathrm{t}}$.

Based on the observations and analyses presented above, a sensible and reasonable indication can be obtained, which assists in determining the termination kinetic parameters. It shows the necessity of including primary termination when modelling non-steady state conditions, particularly for high exposing intensity conditions. In order to accurately estimate a value for $k_{\mathrm{tp}}$, the model is now fit to experimental growth curves of refractive index modulation. A set of growth curves of refractive index modulation were obtained using the same setup as in subsection 3.1 A range of high exposing intensities were examined, i.e., from $10 \mathrm{~mW} / \mathrm{cm}^{2}$ up to $150 \mathrm{~mW} / \mathrm{cm}^{2}$. For clarity and brevity, four typical cases are present. Figure 7 contains the fits to the average experimental index modulation growth curves obtained (from reproducible experimental results) for each of the following exposing intensities: $I_{01}=10 \mathrm{~mW} / \mathrm{cm}^{2}, I_{02}=50 \mathrm{~mW} / \mathrm{cm}^{2}$, $I_{03}=100 \mathrm{~mW} / \mathrm{cm}^{2}$, and $I_{04}=150 \mathrm{~mW} / \mathrm{cm}^{2}$.

We note that for such large exposure intensities the dead band, $t_{\mathrm{i}}$, at the start of grating growth is negligible as can be observed from Figure 7. 


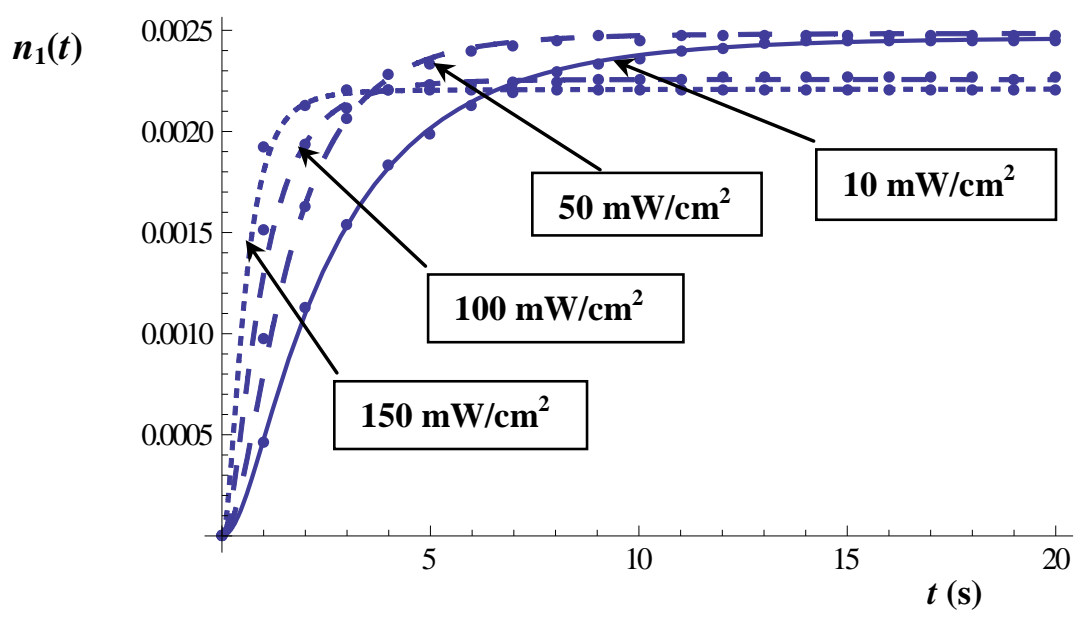

Figure 7. Fit to experimental growth curves (dots) for $I_{0}=: 10 \mathrm{~mW} / \mathrm{cm}^{2}$ (solid curve), $50 \mathrm{~mW} / \mathrm{cm}^{2}$ (long dashed curve), $100 \mathrm{~mW} / \mathrm{cm}^{2}$ (dashed curve), and $150 \mathrm{~mW} / \mathrm{cm}^{2}$ (short dashed curve).

As the exposing intensity increases from $I_{01}=10 \mathrm{~mW} / \mathrm{cm}^{2}$ to $I_{04}=150 \mathrm{~mW} / \mathrm{cm}^{2}$, the rate of the growth of refractive index modulation becomes more rapid, and reaches the saturation, $n_{1 \text { sat }}$, sooner. This trend is mainly ascribed to the highly increased polymerization rate, where monomers are rapidly converted to polymer forming the grating. It is also noted from the experimental results, that further increases of the exposing intensity above $150 \mathrm{~mW} / \mathrm{cm}^{2}$ does not give rise to even faster growth of refractive index modulation. It suggests that the photosensitive dye has reached its absorption limit (absorptivity, $\varepsilon$ ), i.e., the maximum amount of photons the dye molecules can absorb, at a fixed initial dye concentration, $A_{0}$.

These high exposing intensities, not only increase initiation, but also increase the likelihood of primary termination. This results in a shortening of the average polymer chain length and a reduction in the total polymer concentration produced. Thus, as a consequence, there is a reduction in the saturation value of refractive index modulation achieved as can be seen from Figure 7. Table 3 shows the extracted parameters obtained from the fits, along with the corresponding MSE values which indicate the fit quality.

\begin{tabular}{ccccccc}
\hline $\begin{array}{c}\boldsymbol{I}_{\mathbf{0}} \\
\left(\mathrm{mW} / \mathrm{cm}^{2}\right)\end{array}$ & $\begin{array}{c}\boldsymbol{k}_{\mathbf{i}} \\
\left(\mathrm{cm}^{3} / \mathrm{mol} \mathrm{s}\right)\end{array}$ & $\begin{array}{c}\boldsymbol{k}_{\mathrm{p}} \\
\left(\mathrm{cm}^{3} / \mathrm{mol} \mathrm{s}\right) \\
\times 10^{7}\end{array}$ & $\begin{array}{c}\boldsymbol{k}_{\mathrm{t}} \\
\left(\mathrm{cm}^{3} / \mathrm{mol} \mathrm{s}^{7}\right. \\
\times 10^{8}\end{array}$ & $\begin{array}{c}\boldsymbol{k}_{\mathrm{t}} \\
\left(\mathrm{cm}^{3} / \mathrm{mol} \mathrm{s}^{8}\right. \\
\times 10^{10}\end{array}$ & $\begin{array}{c}\boldsymbol{D}_{\mathbf{m}} \\
\left(\mathrm{cm}^{2} / \mathrm{s}\right) \\
\times 10^{-11}\end{array}$ & $\begin{array}{c}\text { MSE } \\
(-) \\
\times 10^{-10}\end{array}$ \\
\hline 10 & 4.86 & 1.57 & 9.09 & 7.05 & 8.93 & 7.17 \\
50 & 4.95 & 1.98 & 6.84 & 8.06 & 7.51 & 3.54 \\
100 & 4.66 & 1.70 & 6.36 & 8.65 & 6.27 & 5.18 \\
150 & 4.98 & 1.79 & 7.42 & 9.18 & 5.76 & 2.23 \\
\hline
\end{tabular}

Table 3. Extracted parameter values from fits to experimental growth curves.

Interestingly the best fits achieved are for values of the primary radical termination rate corresponding to Figure 6(c). Having obtained estimates for the kinetic rate constants which are typical of those presented throughout the literature, predictions about a specific material's behaviour to given experimental conditions can be made. In this way, the necessary laborious experiments required in order to improve a material's performance can be replaced by simple, fast and reliable model simulations. 


\section{REFERENCES}

[1] C. H. Bamford, A. D. Jenkins, R. Johnston, “Termination by primary radicals in vinyl polymerization,” Trans. Faraday Soc. 55(8), 1451-1460, (1959).

[2] C. Decker, B. Elzaouk and D. Decker, "Kinetic study of ultrafast photopolymerizations reactions,” Journal of Macromolecular Science, Pure and Applied Chemistry A33(2), 173-190, (1996).

[3] M. D. Goodner, H. R. Lee, C. N. Bowman, "Method for determining the kinetic parameters in diffusioncontrolled free-radical homopolymerizations," Industrial and Engineering Chemistry Research 36(4), 1247-1252, (1997).

[4] M. D. Goodner and C. N. Bowman, "Modeling primary radical termination and its effects on autoacceleration in photopolymerization kinetics,” Macromolecules 32(20), 6552-6559, (1999).

[5] J. R. Lawrence, F. T. O'Neill, J. T. Sheridan, "Photopolymer holographic recording material," Optik 112(10), 449-463, (2001).

[6] S. Blaya, L. Carretero, R. F. Madrigal, M. Ulibarrena, P. Acebal, A. Fimia, "Photopolymerization model for holographic gratings formation in photopolymers,” Appl. Phys. B-Lasers and Optics 77(6-7), 639-662, (2003).

[7] M. R. Gleeson, J. V. Kelly, D. Sabol, C. E. Close, S. Liu, J. T. Sheridan, "Modelling the photochemical effects present during holographic grating formation in photopolymer materials," J. Appl. Phys. 102(2), 023108, (2007).

[8] M. R. Gleeson, D. Sabol, S. Liu, C. E. Close, J. V. Kelly, J. T. Sheridan, "Improvement of the spatial frequency response of photopolymer materials by modifying polymer chain length,” J. Opt. Soc. Am. B 25(3), 396-406, (2008).

[9] M. R. Gleeson, and J. T. Sheridan, "Non-local photo-polymerization kinetics including multiple termination mechanisms and dark reactions: Part I. Modelling,” J. Opt. Soc. Am. B26(9), 1736-1745, (2009).

[10] M. R. Gleeson, S. Liu, R. R. McLeod, J. T. Sheridan, "Non-local photo-polymerization kinetics including multiple termination mechanisms and dark reactions: Part II. Experimental Validation,” J. Opt. Soc. Am. B26(9), 1746-1754, (2009).

[11] L. Dhar, A. Hale, H. E. Katz, M. L. Schilling, M. G. Schnoes, and F. C. Schilling, "Recording media that exhibit high dynamic range for digital holographic data storage,” Optics Letters 24(7), 487-489, (1999).

[12] S. Schultz, E. Glytsis, T. Gaylord, "Design, fabrication, and performance of preferential-order volume grating waveguide couplers," Applied Optics 39, 1223-1232, (2000).

[13] A. Sato, M. Scepanovic, R. Kostuk, "Holographic edge-illuminated polymer bragg gratings for dense wavelength division optical filters at $1550 \mathrm{~nm}$,” Applied Optics 42, 778-784 (2003).

[14] R. R. McLeod, A. J. Daiber, M. E. McDonald, T. L. Robertson, T. Slagle, S. L. Sochava, L. Hesselink, "Microholographic multilayer optical disk data storage," Applied Optics 44, 3197-3207, (2005).

[15] F. Bruder and T. Faecke, "Materials in Optical Data Storage,” Int. J. Mat. Res. (formerly Z. Metallkd.) 101(2), (2010).

[16] InPhase Technologies, “www.inphase-technologies.com” Tapestry Media, (2007).

[17] M. Gu, M. Straub, L. H. Nguyen, "Complex-shaped 3-D microstructures and photonic crystals generated in a polysiloxane polymer by two-photon microstereolithography,” Opt. Materials, 27, 359-364, (2004).

[18] J. Zhang, K. Kasala, A. Rewari, K. Saravanamuttu, "Self-trapping of spatially and temporally incoherent white light in a photochemical medium,” J. Am. Chem. Soc. 128(2), 406-407, (2006).

[19] G. H. Zhao and P. Mouroulis, "Diffusion-model of hologram formation in dry photopolymer materials," Journal of Modern Optics 41(10), 1929-1939, (1994).

[20] S. Piazzolla and B. K. Jenkins, "Holographic grating formation in photopolymers," Optics Letters 21(14), 10751077, (1996).

[21] V. L. Colvin, R. G. Larson, A. L. Harris, and M. L. Schilling, "Quantitative model of volume hologram formation in photopolymers,” Journal of Applied Physics 81(9), 5913-5923, (1997).

[22] D. J. Lougnot, P. Jost, and L. Lavielle, "Polymers for holographic recording. VI. Some basic ideas for modelling the kinetics of the recording process,” Pure and Applied Optics 6(2), 225-245, (1997).

[23] I. Aubrecht, M. Miler, and I. Koudela, "Recording of holographic diffraction gratings in photopolymers: Theoretical modelling and real-time monitoring of grating growth,” Journal of Modern Optics 45(7), 1465-1477, (1998).

[24] J. H. Kwon, H. C. Hwang, and K. C. Woo, "Analysis of temporal behaviour of beams diffracted by volume gratings formed in photopolymers,” Journal of the Optical Society of America B 16(10), 1651-1657, (1999). 
[25] J. T. Sheridan and J. R. Lawrence, "Nonlocal response diffusion model of holographic recording in photopolymer," Journal of the Optical Society of America A 17(6), 1108-1114, (2000).

[26] C. Neipp, S. Gallego, M. Ortuno, A. Marquez, M. L. Alvarez, A. Belendez, and I. Pascual, "First-harmonic diffusion-based model applied to a polyvinyl-alcohol - acrylamide-based photopolymer,” Journal of the Optical Society of America B 20(10), 2052-2060, (2003).

[27] J. V. Kelly, M. R. Gleeson, C. E. Close, F. T. O'Neill, J. T. Sheridan, S. Gallego, and C. Neipp, “Temporal analysis of grating formation in photopolymer using the nonlocal polymerization-driven diffusion model," Optics Express 13(18), 6990-7004, (2005).

[28] J. T. Sheridan, M. R. Gleeson, C. E. Close, J. V. Kelly, "Optical response of photopolymer materials for holographic data storage applications,” Journal of Nanoscience and Nanotechnology 7(1), 232-242, (2007).

[29] M. R. Gleeson and J. T. Sheridan, "A review of the modelling of free-radical photopolymerisation in the formation of holographic gratings,” J. Opt. A 10, 024008, (2009).

[30] T. Trentler, J. Boyd, V. Colvin, “Epoxy resin photopolymer composites for volume holography,” Chem. Mater. 12, 1431-1438, (2000).

[31] M. R. Gleeson, S. Liu, S. O’Duill, and J. T. Sheridan, "Examination of the photoinitiation processes in photopolymer materials,” J. Appl. Phys. 104, 064917 (2008).

[32] S. Liu, M. R. Gleeson, J. T. Sheridan, "Analysis of the photoabsorptive behaviour of two different photosensitizers in a photopolymer material,” J. Opt. Soc. Am. B 26(3), 528-536, (2009).

[33] S. Liu, M. R. Gleeson, D. Sabol, J. T. Sheridan, "Extended model of the photoinitiation mechanisms in photopolymer materials,” J. Appl. Phys. 106(10), 104911, (2009).

[34] S. Liu, M. R. Gleeson, J. Guo and J. T. Sheridan, "Optical characterization of photopolymers materials: Theoretical and experimental examination of primary radical generation,” Appl, Phys. B, DOI 10.1007/s00340010-4022-x (2010).

[35] L. Carretero, S. Blaya, R. Mallavia, R. F. Madrigal, A. Belendez, and A. Fimia, "Theoretical and experimental study of the bleaching of a dye in a film-polymerization process,” Appl. Opt. 37(20), 4496-4499, (1998).

[36] S. Gallego, M. Ortuno, C. Neipp, A. Marquez, A. Belendez, I. Pascual, J. V. Kelly, and J. T. Sheridan, "Physical and effective optical thickness of holographic diffraction gratings recorded in photopolymers,” Optics Express 13(6), 1939-1947, (2005).

[37] G. Odian, Principles of Polymerization, Wiley, New York, (1991).

[38] M. R. Gleeson, J. V. Kelly, C. E. Close, F. T. O'Neill, and J. T. Sheridan, "Effects of absorption and inhibition during grating formation in photopolymer materials," Journal of the Optical Society of America B 23(10), 20792088, (2006). 